\title{
Tests of Russian multipixel Geiger photodiodes (MRS APD and SiPM) in liquid and gaseous xenon
}

\author{
A. V. Akindinov ${ }^{1}$ \\ Institute for Theoretical and Experimental Physics \\ 117218, Moscow, B. Cheremushkinskaya str. 25, Russia \\ E-mail:Alexander.Akindinov@,cern.ch \\ D. Yu. Akimov, I. S. Alexandrov, A. A. Burenkov, Yu. L. Grishkin, M. V. Danilov, A. \\ G. Kovalenko, V. N. Lebedenko ${ }^{2}$ and V. N. Stekhanov \\ Institute for Theoretical and Experimental Physics \\ 117218, Moscow, B. Cheremushkinskaya str. 25, Russia
}

\begin{abstract}
To evaluate the applicability of multipixel Geiger photodiodes in experiments on dark matter search, such new devices from two Russian manufacturers were studied in liquid and gaseous Xe. Both $n^{+}-p-\pi-p^{++}$("green") and $p^{+}-n-v-n^{++}$("blue") structures have shown photon detection efficiency (PDE) less than $1 \%$ for scintillation radiation at a wavelength of $175 \mathrm{~nm}$. That is not consistent with early data for these devices [1], measured in "self-triggering" mode. Measurements in gaseous xenon have shown the presence of a visible or infrared component and the absence of this component in liquid Xe. That is consistent with early data [2]. Further studies in this field have been performed using wavelength shifting materials and "blue" photodiodes featuring the highest quantum sensitivity in the region of emission of the wavelength shifter. The tests with the use of a wavelength shifter (p-terphenyl) have shown PDE as high as $(10.7 \pm 1.4) \%$.
\end{abstract}

International Workshop on New Photon Detectors (PD09)

Shinshu University Matsumoto Japan

24-26 June 2009

Speaker

Deceased 


\section{Introduction}

In noble gas detectors of Dark Matter particles WIMPs of current generation, the detection of scintillation and electroluminescent is performed by photomultipliers (PMTs). Several experimental groups which develop detectors for Dark Matter experiments are currently investigating semiconductor devices, multipixel avalanche Geiger photodiodes (MGPD; producer's names are: MRS APD (CPTA), SiPM (MEPhI/Pulsar), MPPC (Hamamatsu) and ect).

These photodetectors operate in a single photon counting mode and may replace PMTs in the future because they contribute negligibly low (compared to the PMTs) radioactivity. MRS APD or similar devices are: very compact, low price, low power consuming and low bias voltage (20-70 V). The main disadvantage now for replacement of the PMTs by them is the lack of sensitivity in the VUV region of scintillation and electroluminescent emission of noble gases. The high level of intrinsic thermionic noise of about $1 \mathrm{MHz} / \mathrm{mm}^{2}$ at room temperature seems not to be a big problem since it drops at least by three orders of magnitude operating at the low temperature in liquid noble gases.

The first very promising, but being in contradiction with theory, result of PDE (Photon Detection Efficiency) 5,5\% was obtained by the US group [1] using SiPM in liquid xenon (LXe).

The aim of our work is to prove the possibility to use of MGPD for detection of the LXe scintillation and electroluminescent VUV light.

\section{Existing Russian multipixel avalanche Geiger photodiodes}

The very first Metal-Resistor-Semiconductor APD (MRS APD) was invented in USSR by A. Gasanov, V. Golovin, Z. Sadygov, N. Yusipov (USSR patent \#1702881, from 10/11/1989). The idea was to create a photodiode as an array of microcells assembled on a common silicon substrate and to operate it at supply voltage above the value corresponding to the initiation of a breakdown to reach high gain. During discharge the subsequent voltage drop across the individual resistor (resistive layer) leads to the avalanche quenching and to localization of the discharge within the illuminated microcells, while the rest of the photodiode surface remains in the working state. The main parameters of such "digital" (the total signal is a sum of the pulses with equal amplitude from all fired cell) APD are:

$$
\begin{aligned}
& \mathbf{M}=\mathbf{C}^{*}\left(\mathbf{U}-\mathbf{U}_{\mathrm{br}}\right) \quad \mathbf{Q}=\mathrm{e}^{*} \mathbf{N}_{\text {cells }} * \mathbf{C}^{*}\left(\mathbf{U}-\mathbf{U}_{\mathrm{br}}\right)(\mathbf{1}), \\
& \mathbf{P D E}=\mathbf{Q} . \mathbf{E} . * \mathbf{R}_{\mathrm{G}} * \boldsymbol{\varepsilon}_{\text {geom }}(\mathbf{2}) \text {, }
\end{aligned}
$$

where $\mathbf{M}$ - multiplication, $\mathbf{C}$ - effective cell capacity, $\mathbf{U}$ - bias voltage, $\mathbf{U}_{\mathbf{b r}}$ - breakdown voltage, $\mathbf{Q}$ - charge, $\mathbf{N}_{\text {cells }}$ - number of fired cells, PDE - photon detection efficiency, Q.E. quantum efficiency, $\mathbf{R}_{\mathbf{G}}$ - probability to initiate Geiger discharge from a primary carrier and $\boldsymbol{\varepsilon}_{\text {geom }}$ is a geometrical factor - the ratio of the sensitive area to the full area of the device. Even very first test [3] had shown very good stability of operation, high gain, uniformity of such structure and, as a result, the possibility of single photon detection.

During the last 15 years many efforts were done by three Russian manufactures CPTA, MEPhI/Pulsar and JINR (Dubna)-Zecotek to improve basic characteristics of the photodiodes. 
For instance, CPTA produced MRS APD with trench technology to avoid optical coupling between neighboring cells, when an infrared photon from one cell initiates the new discharge in another. This technology has improved $\boldsymbol{\varepsilon}_{\text {geom }}$ up to $70-75 \%$ and the corresponding PDE up to $40 \%$ [4].

Since the Geiger discharge probability $\mathrm{R}_{\mathrm{G}}$ is higher for electrons than for holes (see related to it ionization coefficient in Fig. 1 up left) and the absorption length in silicon is different for different wavelengths (Fig. 1 up right), there are two currently existing basic structures: $n^{+}-p-\pi-p^{++}$for long wavelengths ("green"; Fig. 1 bottom right) and $p^{+}-n-v-n^{++}$("blue"; Fig. 1 bottom left) for short wavelengths.
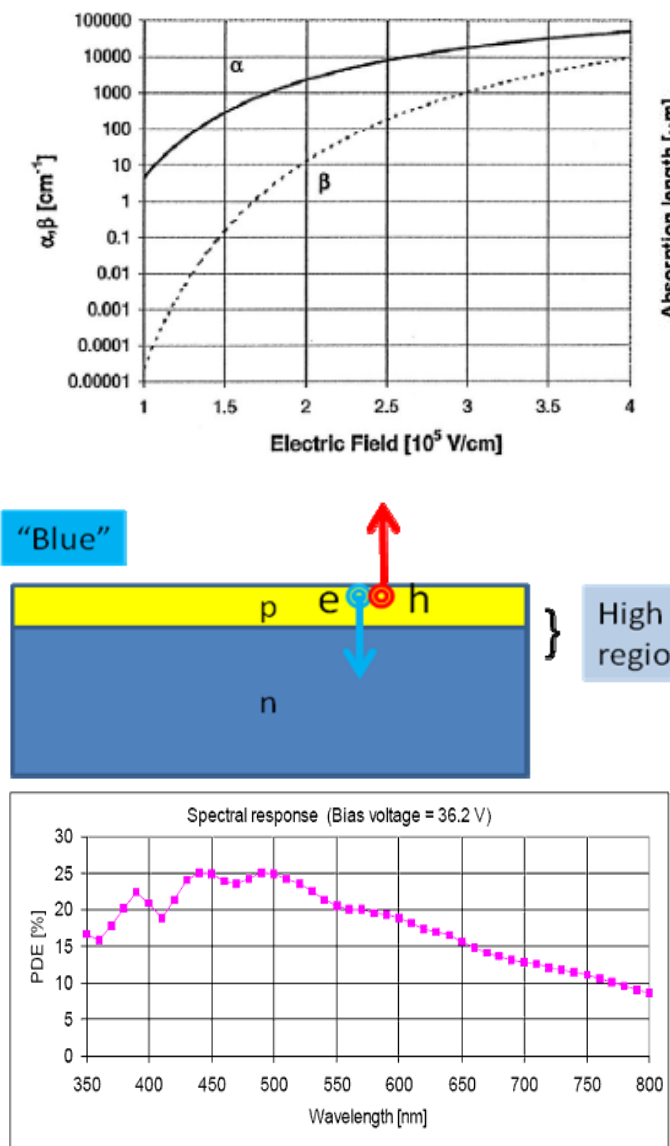

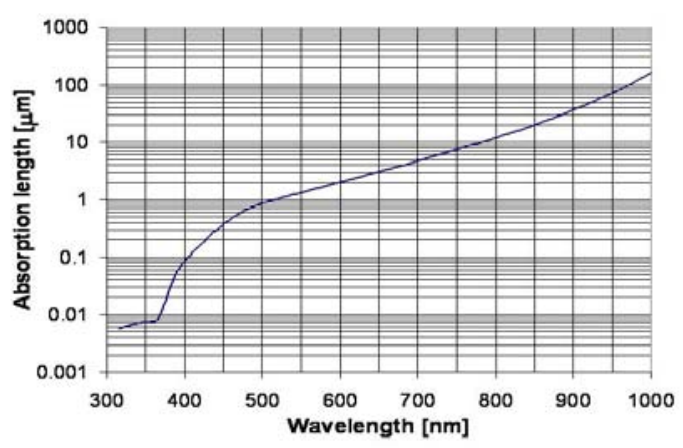

"Green"
High field region

Fig. 1. Ionization coefficient in Si vs. electric field for electrons $(\alpha)$ and holes $(\beta)$ (up

left), absorption length of photons for different wavelength(up right) in Si [5] and typical PDE for CPTA $2 \times 2 \mathrm{~mm}^{2}[6]: p^{+}-n-v-n^{++}$("blue") (down; left) and $n^{+}-p-\pi-p^{++}$ ("green") (down; right)

For our tests we used both the "blue" (Fig. 2 bottom left) and the "green" (Fig. 2 bottom right) CPTA MRS APDs with an active area of $2 \times 2 \mathrm{~mm}^{2}$ (1584 cells) and the "green" structure with an active area of $3 \times 3 \mathrm{~mm}^{2}$ from MEPhI/Pulsar (having PDE of the same shape as that of the "green" CPTA devices, but by a factor of 2 lower) similar to the one used in [1]. All photodiodes were specially manufactured according to the purity requirements using Teflon or metal holders and had bare surface without commonly used epoxy covering. To evaluate the presence of visible or IR light the "green" CPTA photodiode was screened by a window glass which is nontransparent to VUV light. 


\section{Experimental setup}

Measurements were performed in a test chamber filled with liquid or gaseous Xe. An alpha-active ${ }^{241} \mathrm{Am}$ isotope served as a scintillation-light source. The "blue" CPTA photodiode, the "green" photodiode (MEPhI/Pulsar) and the "green" photodiode (CPTA) were placed approximately at equal distances $(\sim 16 \mathrm{~mm})$ from the source; the angles of light incidence on the planes of the photodiodes were also approximately the same. To obtain a trigger signal Hamamatsu R7200 PMT sensitive to $175-\mathrm{nm}$ radiation was mounted in the test chamber. The signals from photodiodes were amplified and, together with the PMT signal, arrived at the inputs of a LeCroy LT344 digital oscilloscope and were written (as waveforms) in a computer for subsequent analysis. More detailed description can be found in [7].
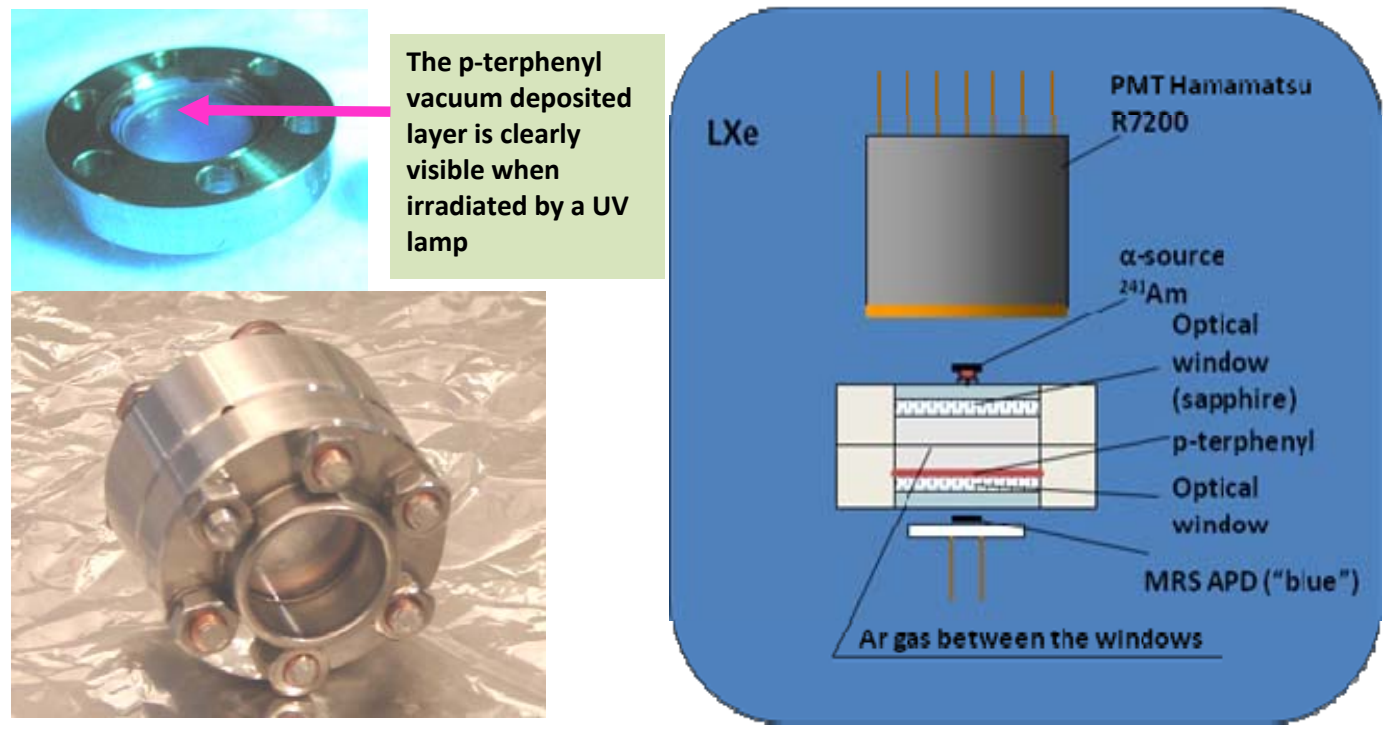

Fig. 2. Photo of 1.33" CF flange with p-terphenyl film deposited on sapphire glass (up left), schematic view of the test assembly (right), and photo of assembly of two windowed flanges (bottom left).

For tests with wavelength (WL) shifter some changes in setup were done. As a WL shifter the vacuum deposited p-terphenyl layer (Fig. 2, right) was used, which was encapsulated in Ar atmosphere between the two windowed (sapphire) 1.33" CF flanges (Fig. 2, right) to prevent Xe pollution. Again PMT Hamamatsu R7200 was used to trigger on the light reemitted by the pterphenyl in backward direction (with respect to the direction of incident light). Only the "blue" photodiode was used in this test. The data recording chain remained the same as in the previous tests.

\section{Results}

In this section we summarized the results of measurements in gaseous and liquid Xe with and without WL shifter.

\subsection{Results for gaseous Xe}

In gaseous $\mathrm{Xe}$, the free path for $\alpha$-particles is about $6 \mathrm{~mm}$. For different trajectories we have different solid angle for light collection. No rigorous estimation of the average solid angle 
for each photodiode has been made, but they are of the same order of magnitude. Using the PMT trigger we observed signals of 1-2 photoelectrons which appear with about the same frequency for both the "blue" and the "green" (screened with glass") photodiodes. This result indicates presence of significant visible or IR component in gaseous Xe. This was proved by other groups [2].

\subsection{Results for Liquid Xe}

In LXe one may assume the light flash is localized in a point since the free path for $\alpha$-particles is negligible in comparison with the distance from the source. PDE can be calculated then as a ratio between the number of photoelectrons $\left(\mathbf{N}_{\text {ph.e. }}\right)$ and the known number of photons from scintillation in corresponding solid angle $\left(\mathbf{N}_{\mathbf{p h}}\right)$ :

$$
\text { PDE }=\mathbf{N}_{\text {ph.e. }} / \mathbf{N}_{\text {ph }} \text {, (4) }
$$

For small signals (of about a few photoelectrons) one can use the following formula to estimate the signal value:

$$
\mathbf{N}_{\text {ph.e. }}=-\ln (\mathbf{P}(0))=-\ln \left(\mathbf{N}_{\text {ped }} / \mathbf{N}_{\text {tot }}\right),(5)
$$

where $\mathbf{N}_{\text {ped }}-$ number of events in pedestal and $\mathbf{N}_{\text {tot }}-$ total number of events. Note that this formula is valid only if the trigger is properly tuned to provide a signal on each light flash. We found PDE $\mathbf{0 . 3 \%}$ for "green" MEPhI/Pulsar SiPM, about 0 \% for "green" CPTA (screened with glass) and $\sim \mathbf{0 . 7 \%}$ for "blue" CPTA diodes respectively. These values are measured for the maximal possible overvoltage about 4.5 V. Measurements were repeated few times during 3 days and shown the same results with 5-7 \% accuracy.

The fact that the photodiode screened with glass have zero PDE indicates the absence of significant visible or IR component in LXe. This is also in agreement with previous data [2].

\subsection{Results for WL shifter in Liquid Xe}

The emission spectrum of LXe and absorption and emission spectra of p-terphenyl together with PDE of "blue" CPTA photodiode are shown in Fig. 3 (up). It is obvious from this graph that only the "blue" structure can be used for detection of the p-terphenyl emission. Fig. 3 (bottom left and right) shows charge spectra with triggering from the PMT and with a random trigger, correspondingly. The latter one is used for single-cell calibration. The $\alpha$-peak position in terms of fired cell number is defined then from this calibration. One must correct this number with the use of the formula:

$$
\mathbf{N}_{\text {ph.e. }}=\mathbf{N}_{\text {cells }} *(1-\alpha),(6)
$$

which takes into account a cross-talk probability $\boldsymbol{\alpha}$, defined as ratio of the 2-nd and 1-st peaks areas in Fig. 3 (bottom right). Since the WL shifter is not deposited directly on a photodiode surface but is spread along a plane located at some distance from it we have mathematically reduced this geometry to a simple case when a WL shifter is on a photodetector surface and the last one spans an equivalent solid angle. Transparency of sapphire window is also taken into account. Finally we have obtained PDE to be $(10.7 \pm 1.4) \%$. 

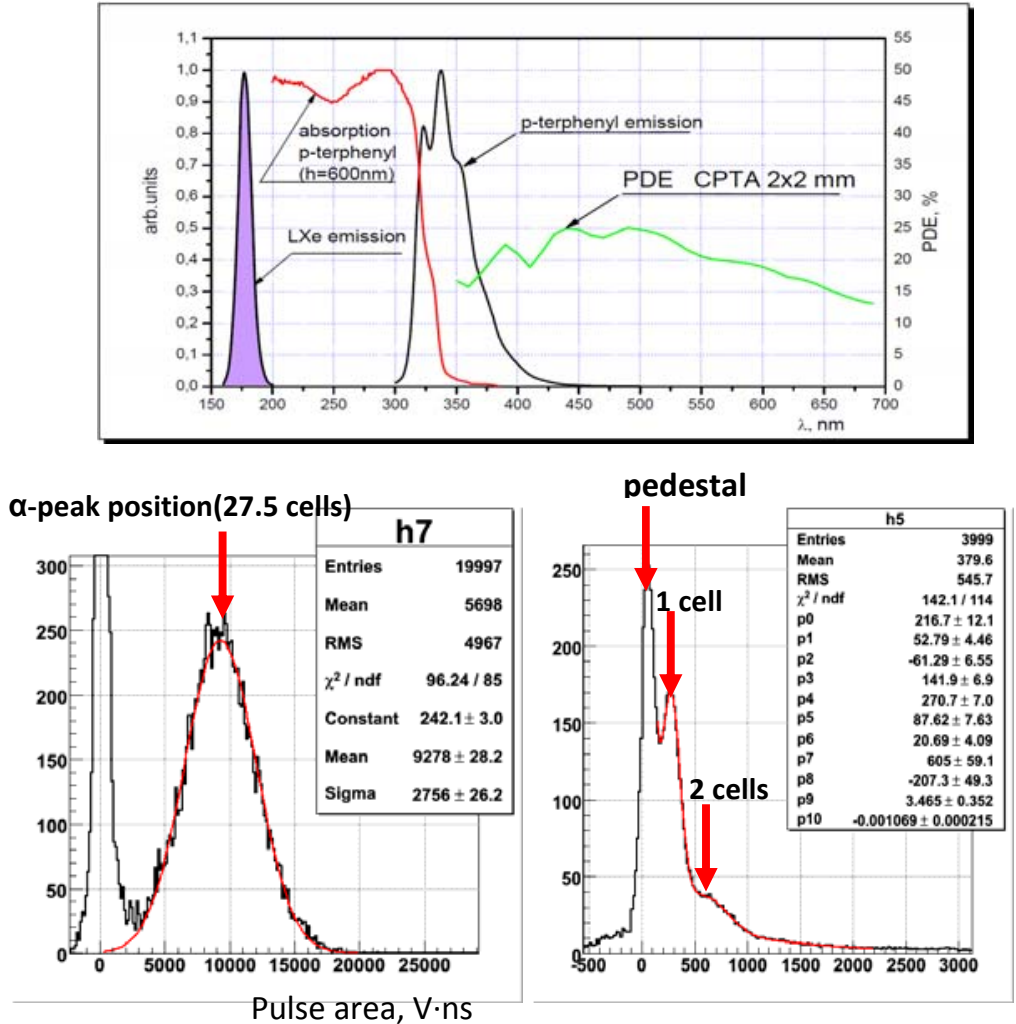

Fig.3. LXe emission and p-terphenyl absorption and emission spectra and PDE of "blue" CPTA photodiode (up; charge spectrum from photodiode: triggered by PMT (bottom left) and with random trigger (bottom right).

\section{Discussion}

All obtained results are in good agreement with prediction and theory, but in contradiction with measurement by US group [1]. We assume that the high PDE value obtained in those measurements may be explained by the fact that they were carried out in a self-triggering mode. In this mode, triggering may be initiated by large area "false" pulses, as in our case when we found (after two circles of cool down and warm up) the presence of about 50-cell signals (Fig. 4) from the SiPM (compare this to the 55-cell peak observed in the US group measurements). We assume that it may be due to a short circuit of individual resistor, hence absence of quenching ("long discharge"), a well known effect explained by CALICE collaboration

\section{Conclusion and plans}

The VUV photon detection efficiencies in LXe were obtained to be less than $1 \%$ for both the "green" and the "blue" structures produced by MEPhI/Pulsar and CPTA. We do not confirm the data on the high VUV detection efficiency presented in [1]. We confirm significant presence of visible or IR light in gaseous Xe.

Further studies have been performed with the use of wavelength-shifting materials and "blue" photodiodes featuring the highest quantum sensitivity in the region of emission of the wavelength shifter. The tests with the use of a WL shifter (p-terphenyl) have shown PDE for 
"blue" CPTA + WL shifter as high as $(10.7 \pm 1.4) \%$. In the future, we plan to use a p-terphenyl protected by poly-para-xylylene which prevents pollution of the WL shifter to LXe. This result will be published soon in a separate article.

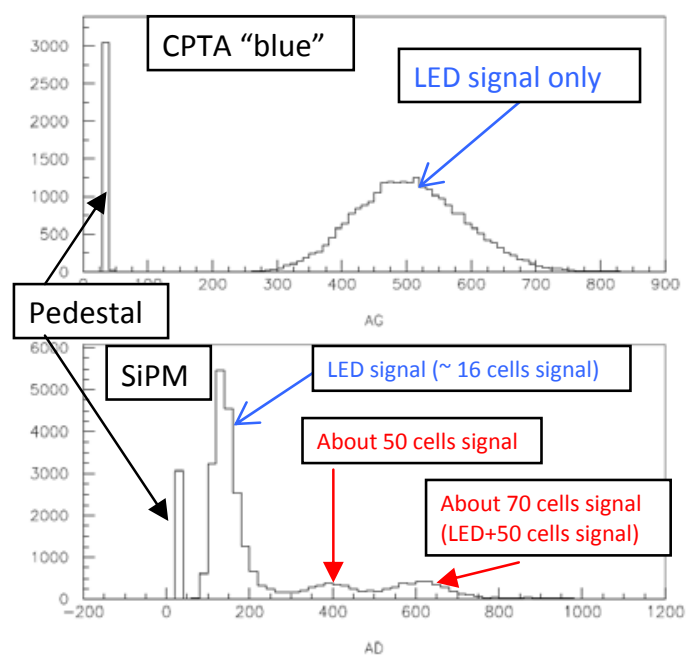

Fig. 4. Charge spectrum for LED flash + about 3000 random triggers for "blue" CPTA and SiPM after cool down and warm up.

\section{Acknowledgments}

We are grateful to the CPTA and in particular to V.M. Golovin for his patience and understanding of difficulties, our colleagues from the MEPhI/Pulsar team for giving us experimental samples, G.B. Bondarenko and Yu. Musienko for useful discussions and Photonique Co. for presented data on the wavelength-dependent detection efficiency for different diodes.

This study was supported in part by the Russian Foundation for Basic Research, projects № 07-02-13569-ofi_ts and 1329.2008.2, and SC Rosatom.

\section{References}

[1]. Aprile E., Cushman P., Ni K., Shagin P., Nucl. Instrum. and Methods Phys. Res. A. 2006. V. 556. P. 215.

[2]. J.A. Wilkerson, J. McLaren, U. Raut, Nucl. Instrum. and Methods Phys. Res. A. 2006. V. 500. P. 345 .

[3]. A.V. Akindinov, A.N. Martemianov, P.A. Polozov, et al., Nucl. Instrum. and Methods Phys. Res. A 387 (1997) 231.

[4]. David McNally and Victor Golovin, Nucl. Instrum. and Methods Phys. Res. A, In Press

[5]. Y. Musienko, private communication

[6]. http://www.photonique.ch/Products_SSPM_main.html\#4.4SSPMS

[7]. D.Yu. Akimov, A.V. Akindinov, A.A. Burenkov et al., Instruments and Experimental Techniques, 2009, Vol. 52, No. 3, pp. 345-351 